\title{
Multiply Guarded Guards in Orthogonal Art Galleries
}

\author{
T.S. Michael ${ }^{1}$ and Val Pinciu ${ }^{2}$ \\ 1 Mathematics Department, United States Naval Academy \\ Annapolis, MD 21402 \\ tsm@nadn.navy.mil \\ 2 Mathematics Department, Southern Connecticut State University \\ New Haven, CT 06515 \\ pinciu@scsu.ctstateu.edu
}

\begin{abstract}
We prove a new theorem for orthogonal art galleries in which the guards must guard one another in addition to guarding the polygonal gallery. A set of points $\mathcal{G}$ in a polygon $P_{n}$ is a $k$-guarded guard set for $P_{n}$ provided that (i) for every point $x$ in $P_{n}$ there exists a point $w$ in $\mathcal{G}$ such that $x$ is visible from $w$; and (ii) every point in $\mathcal{G}$ is visible from at least $k$ other points in $\mathcal{G}$. The polygon $P_{n}$ is orthogonal provided each interior angle is $90^{\circ}$ or $270^{\circ}$. We prove that for $k \geq 1$ and $n \geq 6$ every orthogonal polygon with $n$ sides has a $k$-guarded guard set of cardinality $k\lfloor n / 6\rfloor+\lfloor(n+2) / 6\rfloor$; this bound is best possible. This result extends our recent theorem that treats the case $k=1$.
\end{abstract}

\section{Introduction}

Throughout this paper $P_{n}$ denotes a simple closed polygon with $n$ sides, together with its interior. A point $x$ in $P_{n}$ is visible from point $w$ provided the line segment $w x$ does not intersect the exterior of $P_{n}$. (Every point in $P_{n}$ is visible from itself.) The set of points $\mathcal{G}$ is a guard set for $P_{n}$ provided that for every point $x$ in $P_{n}$ there exists a point $w$ in $\mathcal{G}$ such that $x$ is visible from $w$. Let $g\left(P_{n}\right)$ denote the minimum cardinality of a guard set for $P_{n}$.

A guard set for $P_{n}$ gives the positions of stationary guards who can watch over an art gallery with shape $P_{n}$, and $g\left(P_{n}\right)$ is the minimum number of guards needed to prevent theft from the gallery. Chvátal's celebrated Art Gallery Theorem [1] asserts that among all polygons with $n$ sides $(n \geq 3)$, the maximum value of $g\left(P_{n}\right)$ is $\lfloor n / 3\rfloor$.

Over the years numerous "art gallery problems" have been proposed and studied, in which different restrictions are placed on the shape of the galleries or the powers and responsibilities of the guards. (See the monograph by O'Rourke [7] and the survey by Shermer [8].) For instance, in an orthogonal polygon $P_{n}$ each interior angle is $90^{\circ}$ or $270^{\circ}$, and thus the sides occur in two perpendicular orientations, say, horizontal and vertical. An orthogonal polygon must have an even number of sides. For even $n \geq 4$ we define

$$
g_{\perp}(n)=\max \left\{g\left(P_{n}\right): P_{n} \text { is an orthogonal polygon with } n \text { sides }\right\} .
$$


Kahn, Klawe, and Kleitman [3] gave a formula for $g_{\perp}(n)$ :

Orthogonal Art Gallery Theorem For $n \geq 4$ we have $g_{\perp}(n)=\lfloor n / 4\rfloor$. that

A set of points $\mathcal{G}$ in a polygon $P_{n}$ is a $k$-guarded guard set for $P_{n}$ provided

(i) for every point $x$ in $P_{n}$ there exists a point $w$ in $\mathcal{G}$ such that $x$ is visible from $w$, i.e., $\mathcal{G}$ is a guard set for $P_{n}$; and

(ii) for every point $w$ in $\mathcal{G}$ there are $k$ points in $\mathcal{G}$ different from $w$ from which $w$ is visible.

In our art gallery scenario a $k$-guarded guard set prevents theft from the gallery and prevents the ambush of an insufficiently protected guard. We define the parameter

$$
g g\left(P_{n}, k\right)=\min \left\{|\mathcal{G}|: \mathcal{G} \text { is a } k \text {-guarded guard set for } P_{n}\right\} .
$$

Liaw, Huang, and Lee [4], [5] refer to a 1-guarded guard set for a polygon $P_{n}$ as a weakly cooperative guard set and show that the computation of $g g\left(P_{n}, 1\right)$ is an NP-hard problem. Let

$$
g g_{\perp}(n, k)=\max \left\{g g\left(P_{n}, k\right): P_{n} \text { is an orthogonal polygon with } n \text { sides }\right\} .
$$

The authors [6] have recently determined the function $g g_{\perp}(n, 1)$.

Proposition 1. For $n \geq 6$ we have $g g_{\perp}(n, 1)=\lfloor n / 3\rfloor$.

In this paper we extend Proposition 1 to the "multiply guarded" situations with $k \geq 2$. Here is our main result.

Theorem 1. For $k \geq 1$ and $n \geq 6$ we have

$$
g g_{\perp}(n, k)=k\left\lfloor\frac{n}{6}\right\rfloor+\left\lfloor\frac{n+2}{6}\right\rfloor .
$$

When $k=1$, the expression in (1) simplifies to $\lfloor n / 3\rfloor$ in accordance with Proposition 1,

If $k$ is large, and we require that the guards be posted at vertices of the polygon $P_{n}$, then some vertex must contain more than one guard, that is, the $k$-guarded guard set is actually a multiset. In our proof of Theorem 1] it is convenient to first allow multiple guards at the same vertex ( 55$)$, and then show that the guards can always be moved to distinct points $(\S 6)$.

\section{A Construction}

We begin our proof of Theorem 1 by constructing extremal polygons. Let $P_{n}$ denote the orthogonal polygon of "waves" in Figure 1 The full polygon is used in case $n \equiv 0(\bmod 6)$, while the broken lines indicate the boundaries of a partial 
wave when $n \equiv 2,4(\bmod 6)$. Let $\mathcal{G}$ be a $k$-guarded guard set for $P_{n}$. Each complete wave of $P_{n}$ uses six sides and forces $k+1$ distinct points in $\mathcal{G}$. Also, when $n \equiv 4(\bmod 6)$, the partial wave forces one additional point. Thus $|\mathcal{G}| \geq$ $(k+1)\lfloor n / 6\rfloor$ for $n \not \equiv 4(\bmod 6)$, and $|\mathcal{G}| \geq(k+1)\lfloor n / 6\rfloor+1$ for $n \equiv 4(\bmod 6)$. It follows from some algebraic manipulation that $g g_{\perp}(k, n) \geq|\mathcal{G}| \geq k\lfloor n / 6\rfloor+$ $\lfloor(n+2) / 6\rfloor$ for $n \geq 6$.

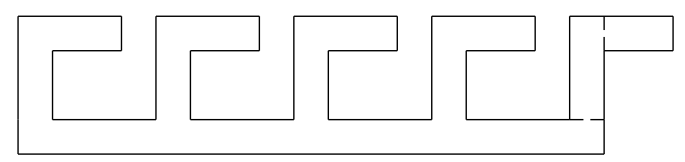

Fig. 1. Orthogonal polygon $P_{n}$ for which $g g\left(P_{n}, k\right)$ is maximum

\section{Galleries, Guards, and Graphs}

Let $P_{n}$ be a simple polygon with $n$ sides. It is well known that diagonals may be inserted in the polygon $P_{n}$ to produce a triangulation, that is, a decomposition of $P_{n}$ into triangles. Diagonals may intersect only at their endpoints. The edge set in a triangulation graph $T_{n}$ consists of pairs of consecutive vertices in $P_{n}$ (the boundary edges) together with the pairs of vertices joined by diagonals (the interior edges) in a fixed triangulation. One readily shows that a triangulation graph is 3-colorable, that is, there exists a map from the vertex set to the color set $\{1,2,3\}$ such that adjacent vertices receive different colors.

Similarly, a quadrangulation $Q_{n}$ of the polygon $P_{n}$ is a decomposition of $P_{n}$ into quadrilaterals by means of diagonals. We refer to $Q_{n}$ as a convex quadrangulation provided each quadrilateral is convex. We also view $Q_{n}$ as a quadrangulation graph in the expected manner. Note that $Q_{n}$ is a plane bipartite graph with an even number of vertices. The (weak) planar dual of $Q_{n}$ is a graph with a vertex for each bounded face of $Q_{n}$, where two vertices are adjacent provided the corresponding faces share an edge. The planar dual of a quadrangulation graph is a tree.

Let $G_{n}=(V, E)$ be a triangulation or quadrangulation graph on $n$ vertices. We say that a set $\mathcal{G}$ of vertices is guard set of $G_{n}$ provided every bounded face of $G_{n}$ contains a vertex in $\mathcal{G}$. If, in addition, every vertex in $\mathcal{G}$ occurs in a bounded face with another vertex in $\mathcal{G}$, then $\mathcal{G}$ is a guarded guard set for $G_{n}$. We let $g\left(G_{n}\right)$ and $g g\left(G_{n}\right)$ denote the minimum cardinality of a guard set and guarded guard set, respectively, for the graph $G_{n}$.

\section{The Proof of Proposition 1: Guarded Guards}

Our proof of Theorem 11 relies on elements contained in our proof [6] of Proposition 1, which we review in this section. The strategy is to employ a coloring 
argument in a triangulation graph as Fisk 2] did in his elegant proof Chvátal's Art Gallery Theorem. Our proof also depends on the following result, which was an important ingredient in the original proof [3] of the Orthogonal Art Gallery Theorem.

\section{Proposition 2. Every orthogonal polygon has a convex quadrangulation.}

The quadrangulation in Proposition 2 may always be selected so that each quadrilateral has positive area, (i.e., its four vertices do not fall on a line), and we shall always do so. However, quadrilaterals with three points on a line are sometimes unavoidable; these degenerate quadrilaterals are an issue in $\S 6$.

The proof of Proposition 1 relies on the following graph-theoretic result.

Proposition 3. We have $g g\left(Q_{n}\right) \leq\lfloor n / 3\rfloor$ for each quadrangulation graph $Q_{n}$ on $n \geq 6$ vertices.

Proof Outline. The proof is illustrated in Figure 2. Let $P_{n}$ be an orthogonal polygon with $n$ sides, and let $Q_{n}$ be the quadrangulation graph for the convex quadrangulation of $P_{n}$ guaranteed by Proposition 2 .

We construct a set $\mathcal{G}$ of vertices in $Q_{n}$ that satisfies (i) $|\mathcal{G}| \leq\lfloor n / 3\rfloor$; (ii) every quadrilateral of $Q_{n}$ contains a vertex of $\mathcal{G}$; (iii) every vertex in $\mathcal{G}$ is contained in a quadrilateral with another vertex in $\mathcal{G}$. Here is our strategy:

- We triangulate $Q_{n}$ by inserting a diagonal in each bounded face to obtain a triangulation graph $T_{n}$ with special properties.

- We 3-color the vertices of $T_{n}$. The least frequently used color gives us a set of vertices $\mathcal{G}^{\prime}$ that satisfies conditions (i) and (ii).

- We shift some vertices of $\mathcal{G}^{\prime}$ along edges of $T_{n}$ to produce a set $\mathcal{G}$ that also satisfies condition (iii).

Triangulate: The graph $Q_{n}$ and its planar dual are both bipartite, and hence we have the vertex biparition $V=V^{+} \cup V^{-}$and the face bipartition $F^{+} \cup F^{-}$ as indicated in Figure 2(a). Each edge of $Q_{n}$ joins a vertex in $V^{+}$and a vertex in $V^{-}$. Each face $f$ of $Q_{n}$ contains two vertices in $V^{+}$and two vertices in $V^{-}$. If $f \in F^{+}$, then we join the two vertices of $f$ in $V^{+}$by an edge, while if $f \in F^{-}$, we join the two vertices of $f$ in $V^{-}$by an edge. The resulting graph is our triangulation $T_{n}$. (See Figure 2(b).) Let $E_{\text {diag }}$ denote the set of edges added to $Q_{n}$ by inserting a diagonal in each face in our triangulation process. Thus our triangulation graph is $T_{n}=\left(V, E \cup E_{\text {diag }}\right)$.

3-Color: We 3-color the triangulation graph $T_{n}$. Let $\mathcal{G}^{\prime}$ be the set of vertices of $T_{n}$ in a color that occurs least frequently. Then $\left|\mathcal{G}^{\prime}\right| \leq\lfloor n / 3\rfloor$; condition (ii) also holds. However, condition (iii) may fail, as in Figure 2(c).

Shift: Let $Y$ denote the set of vertices in $\mathcal{G}^{\prime}$ with degree 3 in $T_{n}$, and let $X$ be the complement of $Y$ in $\mathcal{G}^{\prime}$. Then for each $y \in Y$ there is a unique "conjugate" vertex $y^{*}$ such that $\left[y, y^{*}\right] \in E_{\text {diag }}$. Let $Y^{*}=\left\{y^{*}: y \in Y\right\}$ and define the set $\mathcal{G}=X \cup Y^{*}$.

In [6] we prove that the set $\mathcal{G}$ satisfies conditions (i)-(iii). Thus $\mathcal{G}$ is a guarded guard set for the quadrangulation graph $Q_{n}$, and $|\mathcal{G}| \leq\lfloor n / 3\rfloor$. 


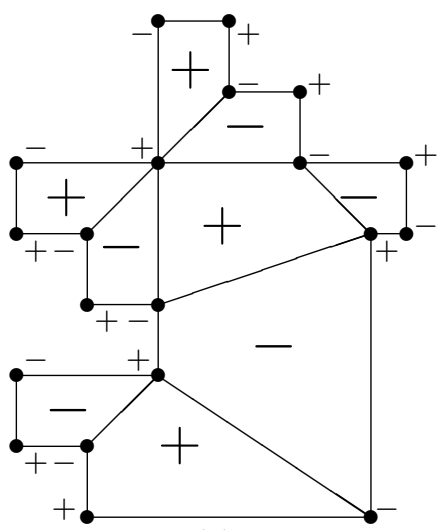

(a)

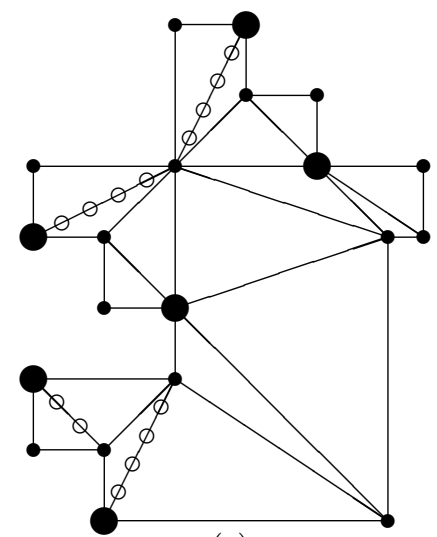

(c)

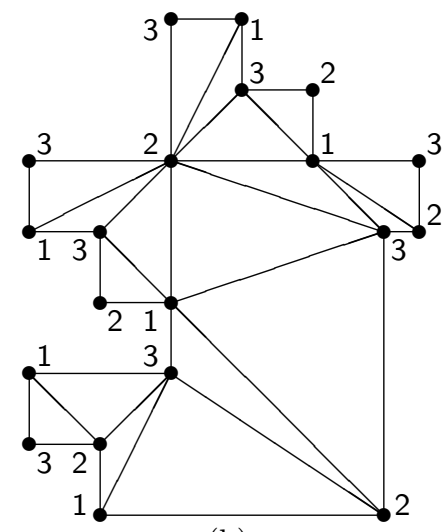

(b)

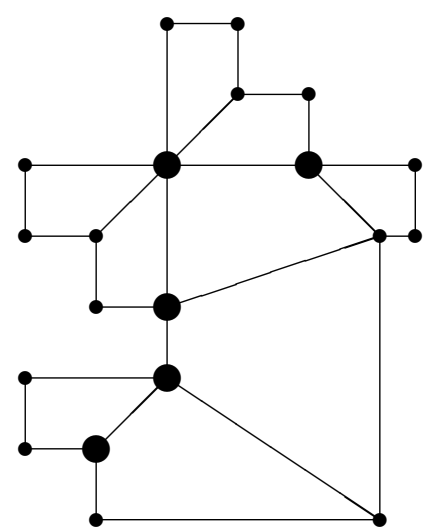

(d)

Fig. 2. The proof of Proposition 1 (a) The quadrangulation graph $Q_{n}$ with vertex and face bipartitions indicated by + and - (b) The triangulation graph $T_{n}$ and a 3-coloring (c) The guard set $\mathcal{G}^{\prime}$; guards in $\mathcal{G}^{\prime}$ at vertices of degree 3 are shifted along the indicated edges (d) The final guarded guard set $\mathcal{G}$ of $Q_{n}$

Now suppose that $P_{n}$ is an orthogonal polygon. Then $P_{n}$ has a convex quadrangulation $Q_{n}$ by Proposition 2 The convexity of the quadrilateral faces implies that the guarded guard set $\mathcal{G}$ in Proposition 3 is a 1-guarded guard set for the orthogonal polygon $P_{n}$. Thus $g g\left(P_{n}, 1\right) \leq\lfloor n / 3\rfloor$. We constructed polygons to establish the reverse inequality in Figure 1, This completes the outline of our proof of Proposition 1

\section{Proof of Theorem 1}

Proposition 1 establishes Theorem 1 for $k=1$. The proof for $k \geq 2$ is illustrated in Figure [3, Let $P_{n}$ be an orthogonal polygon, and let $Q_{n}$ be a convex quad- 


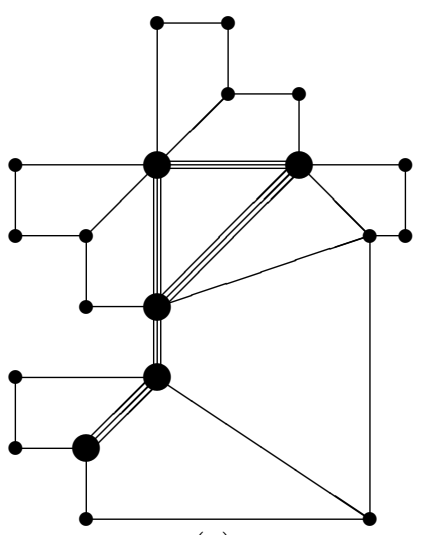

(a)

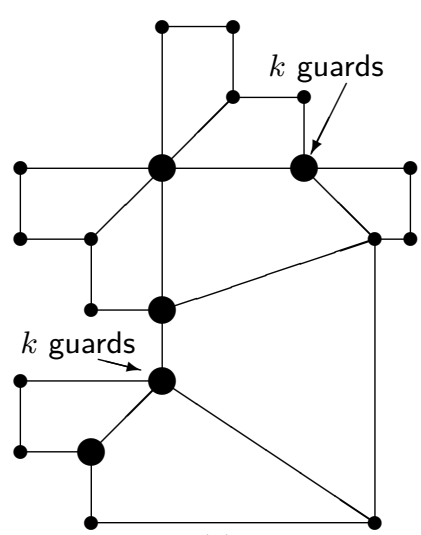

(c)

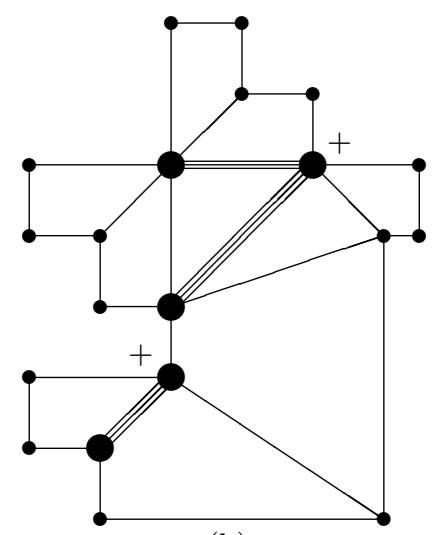

(b)

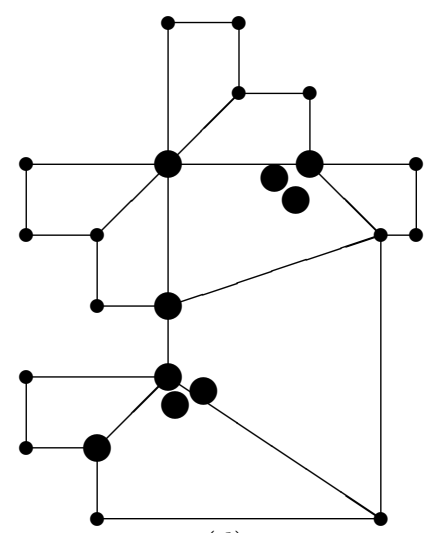

(d)

Fig. 3. The proof of Theorem 1 (a) The guarded guard set $\mathcal{G}$ of the quadrangulation graph $Q_{n}$ from Figure 2 and the graph $G(\mathcal{G})(\mathrm{b})$ A spanning forest of $\operatorname{stars} F(\mathcal{G})$ and a set of centers $\mathcal{G}^{+}$(c) Selection of multiple guards at vertices in $\mathcal{G}^{+}$(d) Separation of multiple guards for $k=3$

rangulation of $P_{n}$. Let $\mathcal{G}$ denote the guarded guard set for the quadrangulation graph $Q_{n}$ produced in the proof of Proposition 1, Now define a graph $G(\mathcal{G})$ whose vertex set is $\mathcal{G}$ with two vertices joined by an edge provided they are both contained in a quadrilateral face of $Q_{n}$. (See Figure 3(a).) No vertex of $G(\mathcal{G})$ is isolated because $\mathcal{G}$ is a guarded guard set of the graph $Q_{n}$. Therefore $G(\mathcal{G})$ has a spanning forest $F(\mathcal{G})$, where each component is a star. (See Figure 3(b).) Let $\mathcal{G}^{+}$be the set of the centers of the stars. (Select either vertex as the center of a star with one edge.) Now $\left|\mathcal{G}^{+}\right| \leq\lfloor|\mathcal{G}| / 2\rfloor$. We insert $k-1$ additional guards at each vertex in $\mathcal{G}^{+}$to obtain a multiset $\mathcal{G}^{*}$ of vertices of $Q_{n}$. Vertices may appear more than once in $\mathcal{G}^{*}$, but this is unavoidable if $k$ is large and we require the guards to be placed at vertices of $Q_{n}$. Now each vertex of $Q_{n}$ is visible from at 
least $k$ others. By Proposition 1 the cardinality of the multiset $\mathcal{G}^{*}$ satisfies

$$
\left|\mathcal{G}^{*}\right|=|\mathcal{G}|+(k-1)\left|\mathcal{G}^{+}\right| \leq\left\lfloor\frac{n}{3}\right\rfloor+(k-1)\left\lfloor\frac{\lfloor n / 3\rfloor}{2}\right\rfloor=k\left\lfloor\frac{n}{6}\right\rfloor+\left\lfloor\frac{n+2}{6}\right\rfloor .
$$

By the convexity of the quadrilateral faces of the orthogonal polygon $P_{n}$, each point in $P_{n}$ is certainly visible from at least one guard, and so we have produced a $k$-guarded guard multiset $\mathcal{G}^{*}$ for $P_{n}$.

\section{Separation of Guards and Degenerate Quadrilaterals}

The $k$-guarded guard multiset $\mathcal{G}^{*}$ constructed in the previous section is satisfactory graph-theoretically, but not geometrically. With the same notation as in the previous section, we now prove that the $k$ guards at each vertex $w$ in $\mathcal{G}^{+}$ can always be separated to obtain a $k$-guarded guard set of points for $P_{n}$, as in Figure 3(d). This is a consequence of the following lemma.

Lemma 1. Let $Q_{n}$ be a convex quadrangulation of the orthogonal polygon $P_{n}$, and let $w$ be a vertex of $P_{n}$. Then there exists a region $R_{w}$ of points in $P_{n}$ such that any vertex in the graph $G(\mathcal{G})$ adjacent to $w$ is visible from every point in $R_{w}$.

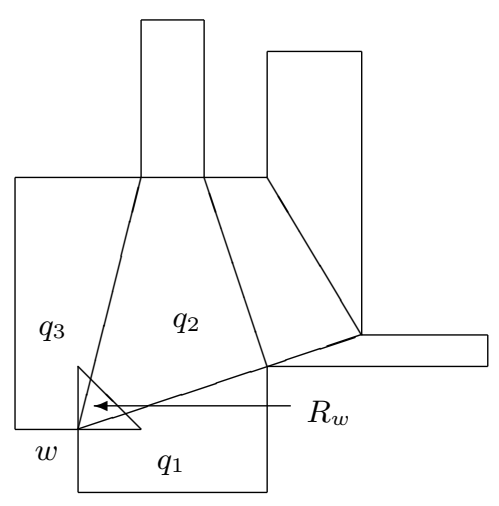

(a)

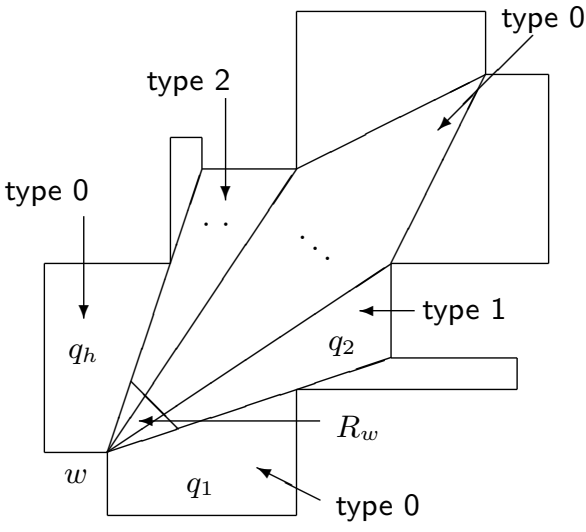

(b)

Fig. 4. The quadrilaterals $q_{1}, q_{2}, \ldots, q_{h}$ at vertex $w$ are all visible from each point in a triangular region $R_{w}$ for both (a) nondegenerate and (b) degenerate quadrilaterals

Proof. The main idea is depicted in Figure 4. If there are no degenerate quadrilaterals at $w$, then a small right triangular region in the "interior quadrant" at $w$ serves as $R_{w}$. When degenerate quadrilaterals are present (with three points on a line), our proof is more complicated, and an acute triangular region serves as $R_{w}$. 
If there is a $90^{\circ}$ angle at $w$, one may readily show that there are no degenerate quadrilaterals at $w$. We now treat the case in which there is a $270^{\circ}$ angle at $w$. Without loss of generality $w$ is at the origin in the Cartesian plane, and $P_{n}$ has edges along the negative $x$ - and $y$-axes. We order the quadrilaterals $q_{1}, q_{2}, \ldots, q_{h}$ that contain $w$ in a counterclockwise manner, as shown in Figure 4. Let $w, x, y, z$ be the vertices in counterclockwise order of a quadrilateral $q$ containing $w$; the interior of $q$ lies to the left as the edges of $q$ are traversed in order. There are three types of quadrilaterals. (See Figure 团)

Type 0: Neither $x$ nor $z$ lies on segment $w y$.

Type 1: Point $x$ lies on segment wy.

Type 2: Point $z$ lies on segment $w y$.

Observation 1: If the point $p$ in $P_{n}$ is in the angle determined by the rays $y x$ and $y z$, then every point in quadrilateral $q$ is visible from $p$.

Now Observation 1 implies that if $p$ is any point in Quadrant I that is sufficiently close to $w$, then every point in a quadrilateral of type 0 is visible from $p$. The degenerate quadrilaterals of types 1 and 2 place further restrictions on our desired set $R_{w}$, which are captured by the following observation.

Observation 2: There exists a nonempty region $R_{w}$ with the desired visibility property provided every quadrilateral of type 1 occurs before the first quadrilateral of type 2 in the list $q_{1}, q_{2}, \ldots, q_{h}$.

We now show that no quadrilateral of type 2 precedes a quadrilateral of type 1, which will complete the proof of the lemma and of Theorem 1. Partition the vertices of $P_{n}$ into the alternating sets $V^{+}$and $V^{-}$, as in the proof of Proposition 1. Without loss of generality $w \in V^{+}$.

Observation 3: In a counterclockwise traversal of the boundary of the polygon $P_{n}$ each vertex in $V^{+}$is entered horizontally and exited vertically, while each vertex in $V^{-}$is entered vertically and exited horizontally.

Claim 1: The line segment $w y$ cannot have negative slope in a quadrilateral $q$ of type 1 or 2. For suppose that vertex $y$ is in Quadrant IV and $q$ is of type 1, as shown in Figure 5(a). Then $x \in V^{-}$, and hence $x$ is entered vertically and is exited horizontally along the boundary of $P_{n}$. But then the interior angle at $x$ must be greater than $270^{\circ}$, which is impossible. The argument is similar when $q$ is of type 2 and when $y$ is in Quadrant II.

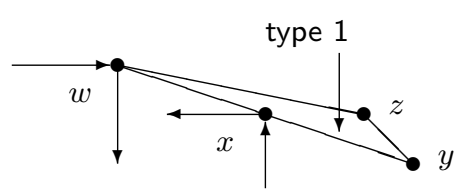

(a)

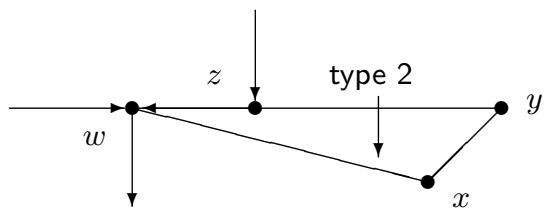

(b)

Fig. 5. (a) The proof of Claim 1 (b) The proof of Claim 2 
Claim 2: Vertices $z$ and $y$ cannot be on the positive $x$-axis in a quadrilateral of type 2. For suppose we have such a quadrilateral, as in Figure 5 b). Then $z \in V^{-}$, and it follows that $z$ is entered from above and is exited to the left. Let $z^{\prime}$ be the point in $V^{-}$along segment $w z$ that is closest to $w$. Then $z^{\prime} w$ must be a boundary edge of $Q_{n}$, and so $w$ meets three boundary edges, which is impossible.

In a similar manner one shows that $x$ and $y$ cannot be on the positive $y$-axis in a quadrilateral of type 1.

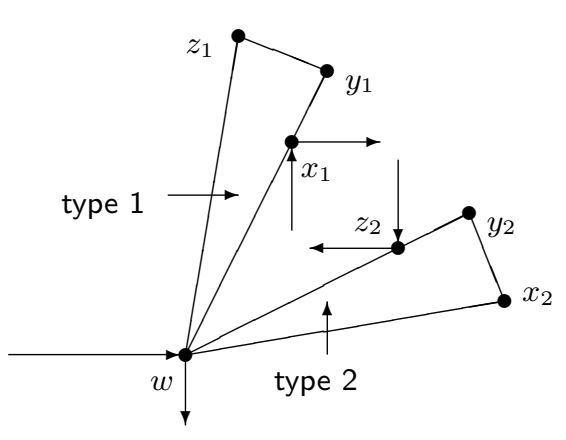

(a)

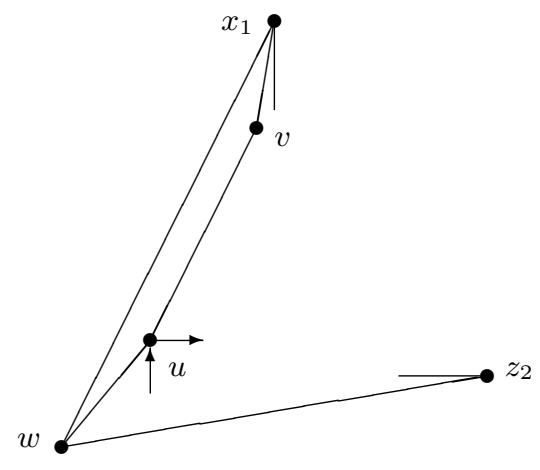

(b)

Fig. 6. (a) A quadrilateral of type 2 cannot precede a quadrilateral of type 1 (b) The proof of Lemma 2

Now assume that a quadrilateral of type 2 with vertices $w, x_{2}, y_{2}, z_{2}$ precedes a quadrilateral of type 1 with vertices $w, x_{1}, y_{1}, z_{1}$ in the list $q_{1}, q_{2}, \ldots, q_{h}$. Then our claims imply that points $y_{1}$ and $y_{2}$ are both in the interior of Quadrant I and that segment $w y_{1}$ is above segment $w y_{2}$, as in Figure 6(a). Also, Observation 3 implies that in a counterclockwise traversal of $P_{n}$ vertex $x_{1}$ must be entered from below and exited to the right, and vertex $z_{2}$ must be entered from above and exited to the left. Now the diagonals $w x_{1}$ and $w z_{2}$ partition $P_{n}$ into three polygons, each of which has a convex quadrangulation. Let $P_{m}$ denote the polygon that has $x_{1}, w$, and $z_{2}$ as consecutive vertices. Then the angles at $x_{1}, w$, and $z_{2}$ in $P_{m}$ must be acute. Thus $P_{m}$ has a convex quadrangulation and each interior angle is either $90^{\circ}$ or $270^{\circ}$, except for the three consecutive acute angles at $x_{1}, w$, and $z_{2}$. The following lemma proves that such a polygon does not exist.

Lemma 2. Let $P_{m}$ be a polygon with each interior angle equal to $90^{\circ}$ or $270^{\circ}$, except for three consecutive acute angles. Then $P_{m}$ does not have a convex quadrangulation.

Proof. Assume that $P_{m}$ does have a convex quadrangulation. We obtain a contradiction by induction. Note that $m$ must be even. Suppose that $m=4$. Then the one non-acute angle of $P_{m}$ must equal $270^{\circ}$, rather than $90^{\circ}$, for the sum of 
the four angles to equal $360^{\circ}$. A quadrilateral with a $270^{\circ}$ angle does not have a convex quadrangulation.

Now suppose that $m \geq 6$. We continue the notation from Lemma 1 and let the three acute angles be at vertices $x_{1}, w$, and $z_{2}$, as in Figure 6 b). We claim that the sum $a$ of these three acute angles must be $90^{\circ}$. For let $P_{m}$ contain $r$ angles equal to $270^{\circ}$. Then $180(m-2)=270 r+(m-3-r) 90+a$, and thus $a=90(m-2 r-1)$. We know that $m$ is even and that $a<270$. The only possibility is $a=90$.

We partition the vertices of $P_{m}$ into two alternating sets $V^{+}$and $V^{-}$, as before, with $w \in V^{+}$, and we orient the edges of $P_{m}$ counterclockwise so that the interior of $P_{m}$ lies to the left of each edge. Each vertex in $V^{-}$is exited horizontally (except for $x_{1}$ ) and is entered vertically (except for $z_{2}$ ). Now let the convex quadrilateral $q$ containing side $x_{1} w$ of $P_{m}$ have vertices $w, u, v, x_{1}$ in counterclockwise order. The sum of the angles in $q$ is $360^{\circ}$, and the angles in $q$ at $w$ and $x_{1}$ sum to less than $90^{\circ}$. Neither of the angles in $q$ at $u$ and $v$ can be greater than $180^{\circ}$. It follows that the angles in $q$ at $u$ and $v$ must be greater than $90^{\circ}$, and therefore the angles at $u$ and $v$ in the polygon $P_{m}$ must equal $270^{\circ}$.

Now $u \in V^{-}$and $u \notin\left\{x_{1}, z_{2}\right\}$. Therefore $u$ is entered vertically and is exited horizontally in a counterclockwise traversal of the boundary of $P_{m}$. The only possibility is that $u$ is entered from below and is exited to the right. Now the diagonal $w u$ partitions $P_{m}$ into two smaller polygons each of which has a convex quadrangulation. One of these smaller polygons contains three consecutive acute angles at $u, w$, and $z_{2}$, with all other angles equal to $90^{\circ}$ or $270^{\circ}$. This contradicts the inductive hypothesis.

\section{References}

1. V. Chvátal, A combinatorial theorem in plane geometry, J. Combin. Theory Ser. B, 18 (1975), 39-41.

2. S. Fisk, A short proof of Chvátal's watchman theorem, J. Combin. Theory Ser. B, 24 (1978), 374.

3. J. Kahn, M. Klawe, and D. Kleitman, Traditional galleries require fewer watchmen, SIAM J. Alg. Disc. Meth., 4 (1983), 194-206.

4. B.-C. Liaw, N.F. Huang, and R.C.T. Lee, The minimum cooperative guards problem on $k$-spiral polygons (Extended Abstract), in Proc. 5-th Canadian Conf. on Computational Geometry (5CCCG), Waterloo, Ontario, Canada, (1993), 97-102.

5. B.-C. Liaw and R.C.T. Lee, An optimal algorithm to solve the minimum weakly cooperative guards problem for 1-spiral polygons, Inform. Process. Lett., 57 (1994), 69-75.

6. T.S. Michael and V. Pinciu, Art gallery theorems for weakly cooperative guards, submitted.

7. J. O'Rourke, Art Gallery Theorems. Oxford University Press, 1987.

8. T.C. Shermer, Recent results in art gallery theorems, Proc. IEEE, 80 (1992), 13841399. 